\title{
Cost-Effectiveness of Dengue Vaccination Programs in Brazil
}

\author{
Eunha Shim ${ }^{1 *}$ \\ ${ }^{1}$ Department of Mathematics, Soongsil University, Seoul, Republic of Korea
}

\begin{abstract}
The first approved dengue vaccine, CYD-TDV, a chimeric, live-attenuated, tetravalent dengue virus vaccine, was recently licensed in 13 countries, including Brazil. In light of recent vaccine approval, we modeled the costeffectiveness of potential vaccination policies mathematically based on data from recent vaccine efficacy trials that indicated that vaccine efficacy was lower in seronegative individuals than in seropositive individuals. In our analysis, we investigated several vaccination programs, including routine vaccination, with various vaccine coverage levels and those with and without large catch-up campaigns. As it is unclear whether the vaccine protects against infection or just against disease, our model incorporated both direct and indirect effects of vaccination. We found that in the presence of vaccine-induced indirect protection, the cost-effectiveness of dengue vaccination decreased with increasing vaccine coverage levels because the marginal returns of herd immunity decreases with vaccine coverage. All routine dengue vaccination programs that we considered were cost-effective, reducing dengue incidence significantly. Specifically, a routine dengue vaccination of 9 -year-olds would be cost-effective when the cost of vaccination per individual is less than \$262. Furthermore, the combination of routine vaccination and large catch-up campaigns resulted in a greater reduction of dengue burden (by up to $93 \%$ ) than routine vaccination alone, making it a cost-effective intervention as long as the cost per course of vaccination is $\$ 255$ or less. Our results show that dengue vaccination would be costeffective in Brazil even with a relatively low vaccine efficacy in seronegative individuals.
\end{abstract}

\section{INTRODUCTION}

Dengue is a febrile illness caused by any one of the four serotypes of dengue virus (DENV-1, DENV-2, DENV-3, or DENV-4). ${ }^{1}$ The disease is transmitted from human to human through the bite of mosquitoes of the genus Aedes. ${ }^{2}$ Dengue is endemic in more than 100 countries, and nearly 4 billion people are at risk for dengue, with 390 million dengue infections occurring every year. ${ }^{3}$

The outcomes of dengue infection range from asymptomatic and subclinical to symptomatic infections. ${ }^{4}$ Symptomatic infections vary from a mild, flu-like illness known as dengue fever (DF) to severe dengue, such as dengue hemorrhagic fever (DHF) and dengue shock syndrome (DSS). ${ }^{2}$ Infection with dengue virus provides long-term protection against the particular serotype that caused the disease. However, dengue infection by one serotype provides only short-lived immunity to the other three dengue virus serotypes. ${ }^{5}$ Furthermore, individuals experiencing their second natural dengue infection have a higher risk of severe disease than those experiencing primary infections, an effect referred to as antibody-dependent enhancement (ADE). ${ }^{6}$

Although six vaccines are in clinical development, to date, only the Sanofi-Pasteur vaccine, Dengvaxia, has completed phase III trials and has been licensed in 13 countries-Mexico, the Philippines, Brazil, El Salvador, Costa Rica, Paraguay, Guatemala, Peru, Indonesia, Thailand, Singapore, Bolivia, and Cambodia. ${ }^{7,8}$ Dengvaxia is a chimeric, live-attenuated, tetravalent dengue virus vaccine (CYD-TDV), based on the licensed yellow fever vaccine, 17D. ${ }^{9}$ A large phase III randomized, controlled vaccine trial of CYD-TDV in Latin America was reported in 2014. ${ }^{10,11}$ The overall efficacy of the vaccine against virologically confirmed dengue cases was $64.7 \%$ (95\% confidence interval $[\mathrm{Cl}]=58.7,69.8)$; however, the efficacy varies depending on serostatus of an individual.

*Address correspondence to Eunha Shim, Department of Mathematics, Soongsil University, 369 Sangdo-Ro, Dongjak-gu, Seoul, Republic of Korea 06978. E-mail: alicia@ssu.ac.kr
Specifically, vaccine efficacy for confirmed cases of dengue was lower in seronegative individuals $(52.5 \%$; $95 \% \mathrm{Cl}=5.9$, 76.1) with $N=387$ for vaccine group and $N=208$ for control group) than in seropositive individuals $(81.9 \% ; 95 \% \mathrm{Cl}=$ $67.2,90.0$ ) with $N=1,560$ for vaccine group and $N=763$ for control group. ${ }^{11-13}$ Furthermore, analysis of the phase III trials of Dengvaxia suggests that there would be an increased risk of hospital admissions that would accompany breakthrough dengue infections in vaccinated seronegative individuals despite the vaccine providing high rates of protection in vaccinated partially dengue-immune individuals (i.e., seropositive). ${ }^{14}$

In light of the recent approval of the administration of CYD-TDV and its variable efficacy, it is essential to consider the cost-effectiveness of dengue vaccination in Brazil. To date, just a few studies on the cost-effectiveness of a hypothetical dengue vaccine have been published. ${ }^{15-21}$ Only one study evaluated the cost-effectiveness of dengue vaccination in Brazil, ${ }^{18}$ whereas another study evaluated the economic burden of dengue in Brazil. ${ }^{22}$ Prior studies predicted that dengue vaccination would be cost-effective up to a total vaccination cost of $\$ 200$ and $\$ 237$ in Thailand and Brazil, respectively. ${ }^{16,18}$ In the Philippines, dengue vaccination was shown to be cost-effective at costs up to $\$ 72$, whereas in Singapore, dengue vaccines would be costeffective under \$53 assuming 10-year vaccine-induced immunity. ${ }^{17,21}$ Although these studies provide valuable evidence that the vaccine would be cost-effective, ${ }^{15-21}$ a substantial amount of additional information has emerged recently, including vaccine safety and efficacy, as well as the target ages of vaccination. This new information has not been addressed in prior cost-effectiveness analyses of dengue vaccination in Brazil. $^{18}$

Herein, we evaluated the cost-effectiveness of dengue vaccination in Brazil, taking into account the reduced vaccine efficacy in seronegative recipients and various vaccination strategies. For this purpose, we used an age-structured model of dengue transmission and vaccination ${ }^{23}$ and fit it to the data on dengue incidence to examine the potential costeffectiveness of deploying a dengue vaccine in Brazil. We first 
estimated the economic and epidemiological impact of dengue vaccination and then calculated its cost-effectiveness at various vaccine costs with and without a catch-up vaccination program. Furthermore, we identified a threshold vaccine cost at which the dengue vaccine becomes cost-effective.

\section{METHODS}

Overview. We considered vaccine interventions using a modified age-structured model of dengue transmission ${ }^{23}$ (Table 1, Supplemental Appendix). Our model reflects the current understanding of the natural history of dengue, and it is a model of the vaccine based on the general results for Dengvaxia. Our model considers the direct effects of vaccination as well as the indirect protective effect of herd immunity for individuals who are not vaccinated.

Dengue infection produces lifelong immunity to the infecting serotype and induces temporary cross-protection against other serotypes, but dengue infection can later enhance disease severity in subsequent infections, due to an effect of ADE. We represent multiple primary, secondary, and tertiary infections with different disease outcome probabilities depending on the number of previous infections. It is known that two prior dengue infections provide protective immunity against severe dengue disease in a subsequent infection. ${ }^{24,25}$ Therefore, our model assumes that third infections from dengue are asymptomatic. ${ }^{24,26-28} \mathrm{We}$ classify infections as asymptomatic ("unapparent") or symptomatic (a "case"). The proportion, $g_{x}$, of infected individuals is assumed to be symptomatic, where the subscript $x$ refers to the epidemiological status of individuals. Symptomatic infections are further separated into mild or severe cases. We assume that the probability of severe disease is dependent on infection history and vaccination status, consistent with empirical data. ${ }^{18,28,29}$ Specifically, we account for ADE by assuming that the probability of severe disease after a secondary infection is greater than after a primary infection. ${ }^{29,30}$ Therefore, our model considers both cross-protection (i.e., no risk of developing a heterotypic infection for a limited time after an infection) and cross-enhancement (i.e., differential risk of developing severe cases on primary, secondary, and tertiary infection). The dengue infection model and the calcu- lation of economic burden associated with dengue infection and vaccination are described in Supplemental Appendix.

Epidemiological parameters. The force of infection is

$$
\lambda_{k}=\frac{\beta_{k} \sum_{k=1}^{6}\left(I_{k}+Y_{k}+J_{k}+\mathrm{VI}_{k}+V Y_{k}\right)}{N}
$$

where $\beta_{k}$ is the age-dependent transmission rate among age group $k$ (Supplemental Figure 1). We also assumed symptomatic and asymptomatic individuals are equally infectious. Our model combines the underlying process of infection in the vector and subsequent transmission to other humans into one aggregate rate, $\beta_{k} \cdot 29$

Cases of dengue in Brazil are known to be substantially underreported. ${ }^{31}$ To fully account for cases of dengue, we ran the model using baseline parameters to equilibrium and calibrated our model to an adjusted annual dengue incidence rate of $2.03 \%$ (including both symptomatic and asymptomatic infections), an annual incidence of DF of $1.07 \%$, and an annual incidence of DHF/DSS of $0.029 \%$, which incorporates underreported cases ${ }^{18,32,33}$. The transmission rates $\left(\beta_{k}\right)$ were chosen to capture the patterns of empirical dengue incidence in Brazil. Specifically, such agespecific incidence profiles (Figure 2) were obtained using $\beta_{k}$ values (Table 2). Therefore, our initial conditions were determined based on the endemic equilibrium of our model. Infected individuals are assumed to recover from primary infections at rate $y$ and gain clinical cross-protection, which prevents clinical illness but allows seroconversion. The average duration of clinical cross-protection is assumed to be $1 / \mathrm{Y}_{C}$ (Table 2).

Vaccine-related parameters. In our model, vaccination reduces the probability of infection with dengue-given exposure, and has no other direct effect on transmission. We assumed that the vaccine efficacy was consistent with the phase III trial results for CYD-TDV in Latin America ${ }^{10,11}$ (Table 2). Trial results indicated that an individual's serostatus before vaccination affects vaccine efficacy. ${ }^{41}$ Specifically, prior dengue infection was shown to increase vaccine efficacy. ${ }^{41,42}$ Such effects were incorporated into our model $(\varepsilon<\delta)$, where we define $\varepsilon$ as vaccine efficacy among

TABLE 1

Model variables

\begin{tabular}{ll}
\hline Symbol & Variable \\
\hline$S_{k}$ & Number of susceptible unvaccinated individuals in age group $k$ \\
$I_{k}$ & Number of primarily infected unvaccinated individuals in age group $k$ \\
$C_{k}$ & Number of unvaccinated individuals recovering from primary infections who are temporarily protected against clinical disease, \\
$R_{k}$ & in age group $k$ \\
$Y_{k}$ & Number of unvaccinated individuals susceptible to secondary infections in age group $k$ \\
$W_{k}$ & Number of unvaccinated individuals with secondary infections in age group $k$ \\
$P_{k}$ & Number of unvaccinated individuals recovering from secondary infections in age group $k$ \\
& in age group $k$ \\
$J_{k}$ & Number of unvaccinated individuals with tertiary infections in age group $k$ \\
$Z_{k}$ & Number of unvaccinated individuals recovering from tertiary infections in age group $k$ \\
$V_{k}$ & Number of partially susceptible vaccinated individuals in age group $k$ \\
$V_{k}$ & Number of primarily infected vaccinated individuals in age group $k$ \\
$V_{k}$ & Number of vaccinated individuals recovering from primary infections and temporarily protected against clinical disease \\
& in age group $k$ \\
$V_{k}$ & Number of vaccinated individuals susceptible to secondary infections in age group $k$ \\
$V_{k}$ & Number of vaccinated individuals with secondary infections in age group $k$ \\
$V_{k}$ & Number of vaccinated individuals recovering from secondary infections in age group $k$ \\
\hline
\end{tabular}


TABLE 2

Epidemiological parameters

\begin{tabular}{|c|c|c|c|}
\hline Symbol & Parameter & Value & References \\
\hline$N_{k}$ & Relative size of age group $k$ & $\begin{array}{c}N_{1}=0.0723, N_{2}=0.0628, N_{3}=0.0157 \\
N_{4}=0.0900, N_{5}=0.0713, N_{6}=0.1082 \\
N_{7}=0.1722, N_{8}=0.1410, N_{9}=0.1884 \\
N_{10}=0.0781\end{array}$ & 53 \\
\hline$b_{k}$ & Birth rate in Brazil in age group $k$ & $\begin{array}{l}b_{1}=3.9616 \times 10^{-5} \\
b_{k}=0 \text { for } k \neq 1\end{array}$ & 53 \\
\hline$p_{k}$ & $\begin{array}{l}\text { Rate of aging out of age } \\
\text { group } k\left(p_{k}=1 / a_{k} \text { where } a_{k} \text { is }\right. \\
\text { the age interval in age group } k)\end{array}$ & $\begin{array}{l}p_{1}=0.0005, p_{2}=0.0007, p_{3}=0.0027 \\
p_{4}=0.0005, p_{5}=0.0007, p_{6}=0.0005 \\
p_{7}=0.0003, p_{8}=0.0003, p_{9}=0.0001 \\
p_{10}=0.0003\end{array}$ & - \\
\hline$\mu_{k}$ & Death rate in age group $k$ & $\begin{array}{l}\mu_{1}=b / N_{1}-p_{1} \\
\mu_{k}=p_{k-1} N_{k-1} / N_{k}-p_{k}(k \neq 1)\end{array}$ & - \\
\hline$\beta_{k}$ & $\begin{array}{l}\text { Transmission rate among } \\
\text { age group } k\end{array}$ & $\begin{array}{l}\beta_{1}=0.1256, \beta_{2}=0.1209, \beta_{3}=0.1302 \\
\beta_{4}=0.1488, \beta_{5}=0.1953, \beta_{6}=0.1767 \\
\beta_{7}=\beta_{8}=0.1860, \beta_{9}=\beta_{10}=0.2325 \\
\beta_{11}=\beta_{12}=\beta_{13}=\beta_{14}=0.1860, \beta_{15}=0.2418\end{array}$ & Data fitting \\
\hline$\sigma_{n}$ & $\begin{array}{l}\text { Relative probability of being } \\
\text { susceptible to } n \text {th infection }\end{array}$ & $(5-n) / 4$ & 18 \\
\hline$\phi_{k}$ & Vaccination rate in age group $k$ & $\begin{array}{l}\phi_{3}=0.00174 \text { and } \phi_{k}=0 \text { for } k \neq 3 \text { for } \\
\text { Strategy } A \\
\phi_{3}=\phi_{4}=0.00174 \text { and } \phi_{k}=0 \text { for } k \neq 3 \text { or } 4 \\
\text { for Strategy B }\end{array}$ & Author's assumption \\
\hline$z_{R}$ & $\begin{array}{l}\text { Wastage rate for routine } \\
\text { vaccination program }\end{array}$ & $10 \%$ & 34,36 \\
\hline$z_{C}$ & Wastage rate for catch-up campaign & $5 \%$ & 34,36 \\
\hline$\varepsilon$ & $\begin{array}{l}\text { Vaccine efficacy against infection } \\
\text { among the seronegative aged } \\
\text { nine and over }\end{array}$ & 0.616 & 12 \\
\hline$\delta$ & $\begin{array}{l}\text { Vaccine efficacy against infection } \\
\text { among the seropositive aged } \\
\text { nine and over }\end{array}$ & 0.792 & 12 \\
\hline \multirow[t]{2}{*}{$g_{x}$} & $\begin{array}{l}\text { Proportion of dengue infections that } \\
\text { are symptomatic in the } \\
\text { epidemiological class } x_{k}\end{array}$ & 0.45 for $x_{k}=I_{k}$ & 8 \\
\hline & & $\begin{array}{l}0.8 \text { for } x_{k}=Y_{k} \text { or } V I_{k} \\
0.14 \text { for } x_{k}=J_{k} \text { or } V Y_{k}\end{array}$ & \\
\hline$y$ & $\begin{array}{l}\text { Fraction of DF cases that sought } \\
\text { medical care }\end{array}$ & 0.5 & 37,38 \\
\hline Y & Rate of recovery from infection & $0.146 /$ day & 30 \\
\hline$Y_{C}$ & Rate of loss of cross-immunity & $0.0055 /$ day & 35,39 \\
\hline$h_{x}$ & $\begin{array}{l}\text { Probability of developing DHF/DSS } \\
\text { after symptomatic infection among } \\
\text { the individuals in the } \\
\text { epidemiological class } x_{k}\end{array}$ & 0.045 for $x_{k}=Y_{k}$ or $\mathrm{VI}_{k}$ & 8 \\
\hline $\mathrm{X}$ & Risk of death from DHF/DSS & $\begin{array}{l}0.25 h_{Y} \text { for } x_{k}=I_{k}, J_{k} \text { or } \mathrm{VY}_{k} \\
0.01\end{array}$ & 18,40 \\
\hline
\end{tabular}

individuals aged nine and over who had never been exposed to dengue (referred to as seronegative individuals), whereas $\delta$ denotes the vaccine efficacy among individuals aged nine and over who had previously been exposed dengue virus (referred to as seropositive individuals).

Furthermore, our model incorporates not only vaccineinduced protection but also vaccine-enhanced dengue disease among vaccine recipients, as observed in the CYD-TDV trials. $^{10,24}$ Results of phase III efficacy trials of CYD-TDV conducted in Latin America demonstrated that vaccination may present immunological similarities to an attenuated subclinical primary infection; thus, vaccination of seronegative individuals potentially increases the risk of DHF during a subsequent wild-type infection. ${ }^{41}$ Therefore, in our model, the probability of developing DHF/DSS after primary symptom- atic infection among unvaccinated individuals was assumed to be lower than individuals who were seronegative when vaccinated $\left(q_{l}<q_{\mathrm{VI}}\right)$. Here, $q_{\mathrm{l}}$ and $q_{\mathrm{vl}}$ are defined as the probability of developing DHF among symptomatically infected individuals in $I_{k}$ and $\mathrm{VI}_{k}$, respectively (Table 2 ).

Vaccination policies considered. In prior studies, many national vaccination strategies were tested: routine vaccination at various ages, including 9-year-olds, considering reports of pooled vaccine efficacy for 9-16 years of age, ${ }^{12}$ and catch-up immunization campaigns for various age groups. ${ }^{13,34}$ In our paper, the default policy was routine vaccination of 9 -year-old children, the youngest age within the approved age range (9-45 years) for the Dengvaxia, with $70 \%$ vaccine coverage level. For sensitivity analysis, we examined the impact of lower (50\%) and higher $(90 \%)$ 
TABLE 3

Probabilities and costs of dengue infection

\begin{tabular}{lllll}
\hline & Probability & $\begin{array}{c}\text { Relative } \\
\text { probability }\end{array}$ & $\begin{array}{c}\text { Direct } \\
\text { costs (\$) }\end{array}$ & References \\
\hline $\begin{array}{l}\text { Dengue infection in the } \\
\text { epidemiological class } x_{k}\end{array}$ & 1.00 & & & \\
Asymptomatic & $1-g_{x}$ & & & 3 \\
Symptomatic & $g_{x}$ & 1.00 & & 3 \\
DF & & $1-q_{x}$ & & $18,28,29$ \\
Ambulatory & & $Y\left(1-q_{x}\right)$ & 72 & 43 \\
Severe (DHF) & $q_{x}$ & & $18,28,29$ \\
Hospitalized & $(1-\mathrm{X}) q_{x}$ & 267 & 43 \\
Death & $\mathrm{X} q_{x}$ & $\mathrm{NA}$ & 43 \\
\hline
\end{tabular}

DF = dengue fever; $\mathrm{DHF}=$ dengue hemorrhagic fever; $\mathrm{NA}$ = non applicable; all values are reported in 2017 U.S. dollars

vaccine coverage levels with routine vaccination in terms of cost-effectiveness. Alternative strategies examined were 1 year of catch-up campaigns for various age groups followed by routine vaccination of 9 -year-olds. For catch-up campaigns, we considered ages 10-18, 10-25, 10-35, and $10-45$ as the targeted ages. For all catch-up campaigns considered, it was assumed that $70 \%$ of 9 -year-olds received routine vaccination and $50 \%$ vaccine coverage was achieved in the catch-up campaigns.

Costs. To calculate costs, we assumed that a fraction of DF patients seek medical care, requiring ambulatory care (Table 3). For DHF cases, we assumed that hospitalization was required. The probability of developing DHF/DSS after symptomatic infections is assumed to be dependent on the serostatus and the vaccination status of each individual.

Economic and health outcomes. We identified many epidemiological and economic parameters from the literature and publicly available data (Tables 2 and 4). Using those parameters, we evaluated the epidemiological impact of vaccination strategies by contrasting the projected dengue burden with and without vaccine deployment over a 10-year forecasted period. To calculate health outcomes, we calculated the time-discounted disability-adjusted life-years (DALYs) lost to DF, DHF/DSS, and dengue-related deaths. Our estimated health impact is presented in quality-adjusted life-years (QALYs), by assuming that one DALY averted is equivalent to one QALY gained, as in previous studies. ${ }^{49-51}$

We calculated the total costs accrued due to vaccination and medical treatment. These monetary costs were calculated in 2017 U.S. dollars based on the Consumer Price Index for Medical Costs. ${ }^{52}$ To calculate the incremental costeffectiveness ratios from a health-care perspective, we included direct medical costs and health outcomes and used an annual discount rate of $3 \%$ over a 10 -year period. ${ }^{53}$

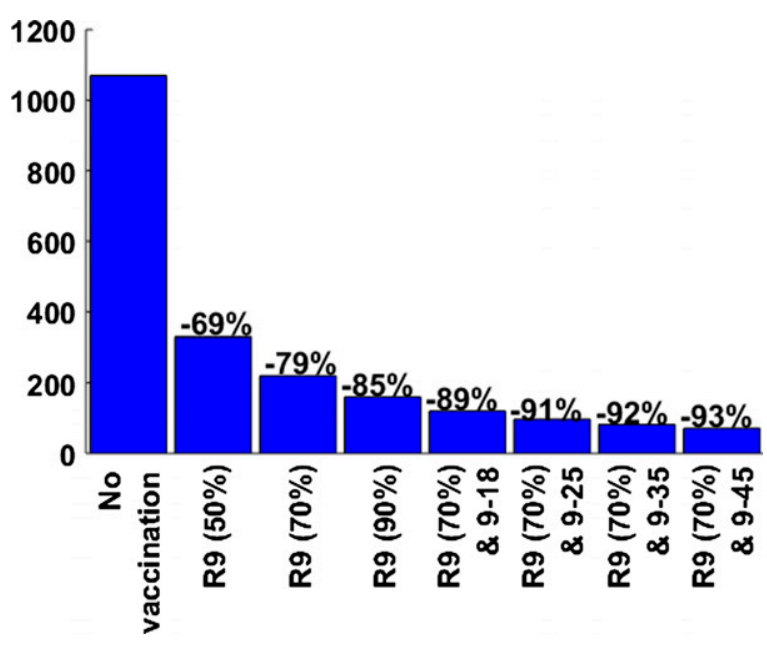

FIGURE 1. Expected yearly incidence of symptomatic cases of dengue per 100,000 for the different vaccination strategies. Incidence was averaged over a 10 -year period. Percentage values refer to the percent reduction in dengue cases compared with dengue incidence in the prevaccine era. Each vaccination strategy is indicated by the age of routine vaccination with its coverage levels and the target ages in the catch-up campaign. For example, R9 (70\%) and 9-25 refer to a catch-up campaign of individuals from 9- to 25 -year-olds followed by routine vaccination at 9-year-olds with a coverage level of $70 \%$. For all catch-up campaigns considered, $50 \%$ vaccine coverage was assumed.

Cost-effectiveness of vaccination policies. To determine the net QALYs gained, we subtracted the total QALYs lost across the population under the vaccination scenario from the total QALYs lost across the population under the no-vaccination scenario (see Supplemental Appendix). To determine the net costs accrued, we subtracted the total costs accrued under the no-vaccination scenario from the total costs accrued under the vaccination scenario. Because both vaccine procurement and delivery costs were unknown, we varied the vaccination cost, including the cost of vaccine doses, the costs of vaccine delivery and administration, and the cost associated with vaccine wastage.

The Brazilian guidelines for health technology assessments do not specify a threshold to determine whether an intervention is cost-effective, and thus we use the threshold derived from the World Health Organization Commission on Macroeconomics and Health. ${ }^{54}$ In addition, we assumed that society's willingness to pay (WTP) for one DALY averted is equivalent to its WTP for one QALY, as in previous studies. ${ }^{49-51}$ Therefore, in our analysis, we assumed that interventions that

TABLE 4

Cost-effectiveness parameters

\begin{tabular}{lll}
\hline Symbol & \multicolumn{1}{c}{ Parameter } & \multicolumn{1}{c}{ Value } \\
\hline$r$ & Social discount rate for QALYs calculations & 0.03 \\
$D_{\text {Death }}$ & Disability weight for death & 1 \\
$D_{\mathrm{DF}}$ & Disability weight for DF & 0.197 \\
$D_{\mathrm{DHF}}$ & Disability weight for DHF/DSS & 0.545 \\
$L_{\mathrm{DF}}$ & Time lost due to DF (years) & 0.019 \\
$L_{\mathrm{DHF}}$ & Time lost due to DHF/DSS (years) & 0.0325 \\
$L_{\text {Death, } k}$ & Years of life lost due to death for age group $k$ & 67.5 for $k=1,63$ for $k=2$ \\
& & 61 for $k=3,57.5-5(k-4)$ for $k=4, \ldots, 15$ \\
$a_{k}$ & Average age of dengue exposure in age class $k$ & 2.5 for $k=1,7$ for $k=2$, \\
& & 9 for $k=3,5(k-4)+12.5$ for $k=4, \ldots, 15$ \\
\hline
\end{tabular}

DF = dengue fever; DHF = dengue hemorrhagic fever; DSS = dengue shock syndrome; QALY = quality-adjusted life-years. 


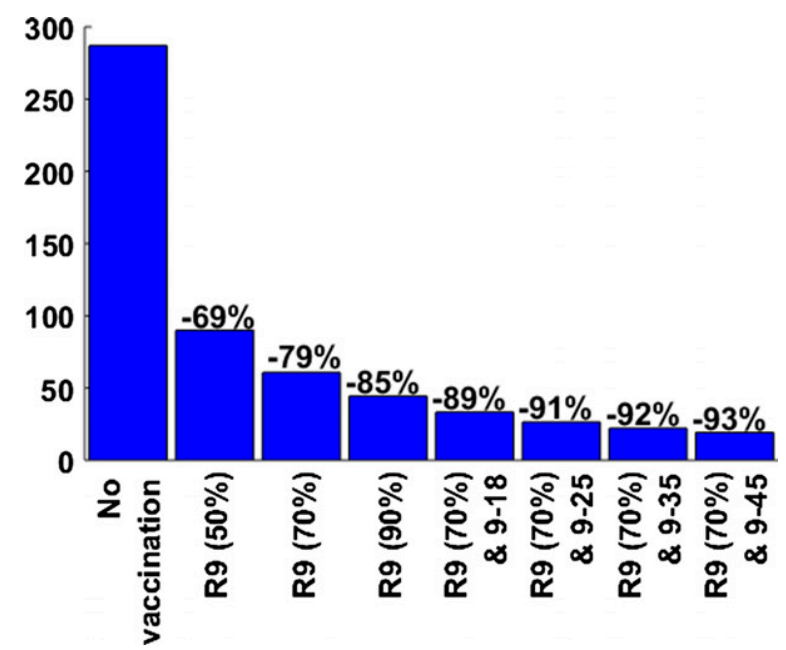

FIGURE 2. Expected yearly incidence of dengue hemorrhagic fever per million for the different vaccination strategies. Incidence was averaged over a 10-year period. Percentage values refer to the percent reduction in dengue cases compared with dengue incidence in the prevaccine era. Each vaccination strategy is indicated by the age of routine vaccination with its coverage levels and the target ages in the catch-up campaign. For example, R9 (70\%) and 9-25 refers to a catch-up campaign of individuals from 9- to 25-year-olds followed by routine vaccination at 9 -year-olds with a coverage level of $70 \%$. For all catch-up campaigns considered, $50 \%$ vaccine coverage was assumed.

would gain one additional QALY for less than three times the average per capita gross domestic product (GDP), \$25,620, were deemed "cost-effective," and those that would gain one additional QALY for less than the average per capita GDP $(\$ 8,540)$ were deemed "very cost-effective" $" 54,55$. The costs and utility weights used in the analysis are presented in Table 4.

\section{RESULTS}

Our calculated values for the annual dengue infection incidence in the absence of vaccination (2.03\%) and the annual symptomatic DF incidence (1.07\%) were comparable to empirical estimates (Figures 1 and 2). ${ }^{18,35,43}$ The transmission rates $\left(\beta_{k}\right)$ were chosen to capture the age distribution among the cases of dengue in Brazil; the simulated age distributions of symptomatic cases of dengue in the prevaccine era are presented (Figure 3). Before the dengue vaccine was introduced, the annual DHF incidence in Brazil was estimated to be $0.029 \%$, resulting in a total cost of dengue at $\$ 906$ million to the health-care system.

Our model shows, however, that the epidemiological burden associated with dengue would be significantly reduced by vaccination (Figures 1 and 2). Figure 1 presents the 10-year impact of each vaccination scenario in terms of the percent reduction of all symptomatic cases of dengue. The reduction in the incidence of dengue would range from $69 \%$ with a routine vaccination of 9 -year-olds and $50 \%$ vaccine coverage to $93 \%$ with one year of a catch-up campaign targeting 9 - to 45 -year-olds with $50 \%$ vaccine coverage followed by a routine vaccination at 9 -year-olds. This means that the number of dengue cases prevented over a 10-year period compared with the situation without vaccination would range from $14,800,000$ with routine vaccination of 9 -year-olds $(50 \%$ vaccine coverage) to $19,979,900$ with a catch-up campaign of 9- to 45-year-olds followed by routine vaccination of 9 -year-olds (70\% vaccine coverage level). Similarly, the number of DHF cases prevented over a 10-year period with a vaccination program is estimated to range from 394,024 with routine vaccination of 9 -year-olds $(50 \%$ vaccine coverage level) to 535,660 with a catch-up campaign for 9- to 45 -year-olds followed by routine vaccination of 9 -year-olds (70\% vaccine coverage).

In addition, we examined the age-specific impact of dengue vaccination with respect to the age distributions of DF and DHF cases in the pre- and postvaccine eras (Figure 3 and 4). We found that after 10 years of routine vaccination of 9-year-olds, $14 \%$ of DF cases would occur among individuals aged $9-18$, but in the prevaccine era, $18 \%$ of DF cases occurred in the same age group. However, the relative incidence of DHF increased by $1 \%$ in 9 - to 18 -year-olds and those over 36-year-olds.

We evaluated the vaccine programs' cost-effectiveness with increasing cost per course of vaccination (Figure 5 and 6). In general, dengue vaccination in Brazil was more costeffective with lower vaccine coverage levels. Specifically, with $50 \%$ vaccine coverage for the routine vaccination
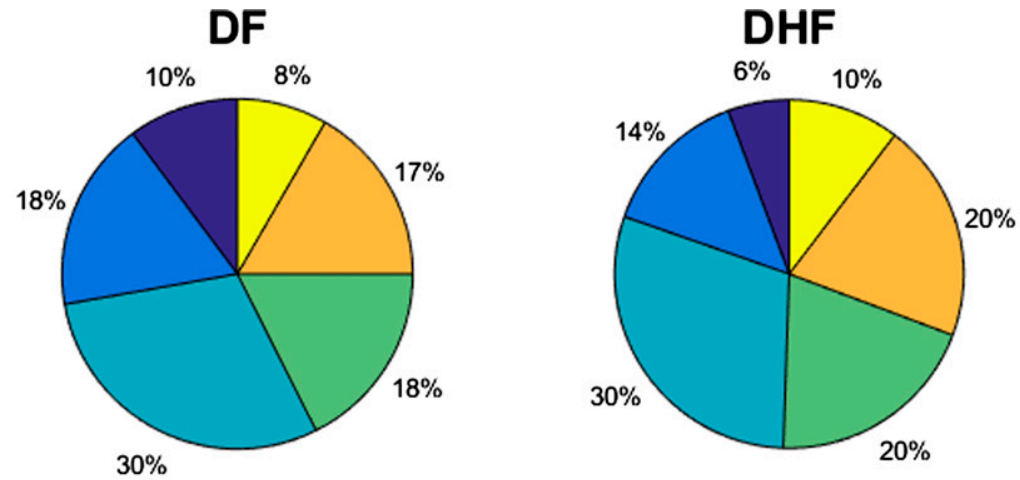

$\square$ Age $<9 \square$ Age 9-18 $\square$ Age 19-35

Age $36-45$

Age 46-64

Age 65+

FiguRE 3. Age distributions of dengue fever (DF) and dengue hemorrhagic fever (DHF) cases in the prevaccine era. Age-specific incidence rates of DF and DHF cases in the prevaccine era are presented. An annual incidence of dengue fever and DHF cases are $1.07 \%$ and $0.029 \%$, respectively. 

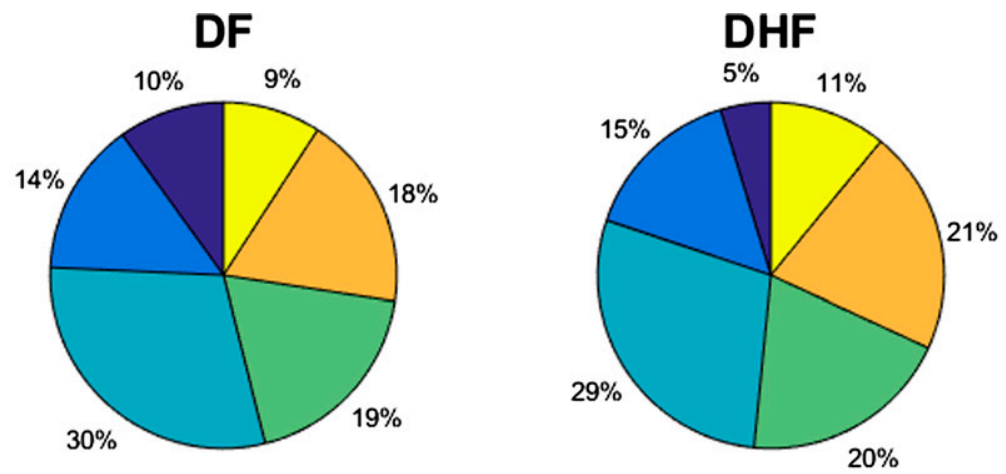

$\square$ Age $<9$

Age 9-18

Age 19-35

Age 36-45

Age 46-64

Age 65+

FIGURE 4. Age distributions of dengue fever (DF) and dengue hemorrhagic fever (DHF) cases after a 10-year period when routine vaccination of 9-year-olds are in practice. After 10 years of routine vaccination of 9-year-olds, the relative incidence of DF decreased among 9- to 18-year-olds by $4 \%$, whereas it increased among individuals over 36 -year-olds. The relative incidence of DHF increased by $1 \%$ in 9 - to 18 -year-olds and those over 36-year-olds.

program, the cost per QALY gained from the health-care perspective is less than the $\$ 11,208$ GDP per capita in Brazil when the cost of vaccination is $\$ 100$ or lower, making the dengue vaccination program a very cost-effective intervention (Figure 5). Such a threshold cost of dengue vaccination per person, which allows the vaccination program to be very cost-effective, increases to $\$ 130$ and to $\$ 160$, when the vaccine coverage level increases to $70 \%$ and $90 \%$, respectively. The threshold price per course of vaccination for which the cost of vaccination equaled three times the GDP is \$262 for routine vaccination of 9 -year-olds for $90 \%$ vaccine coverage. Therefore, a routine dengue vaccination of 9-year-olds would be cost-effective when the cost of vaccination per individual is $<\$ 262$ for a vaccine coverage level of $90 \%$ or lower. For catch-up campaigns followed by routine vaccination, the cost-effectiveness decreases with wider target age groups (Figure 6). At a vaccination cost of $\$ 255$ or lower, all catch-up campaigns considered were cost-effective.

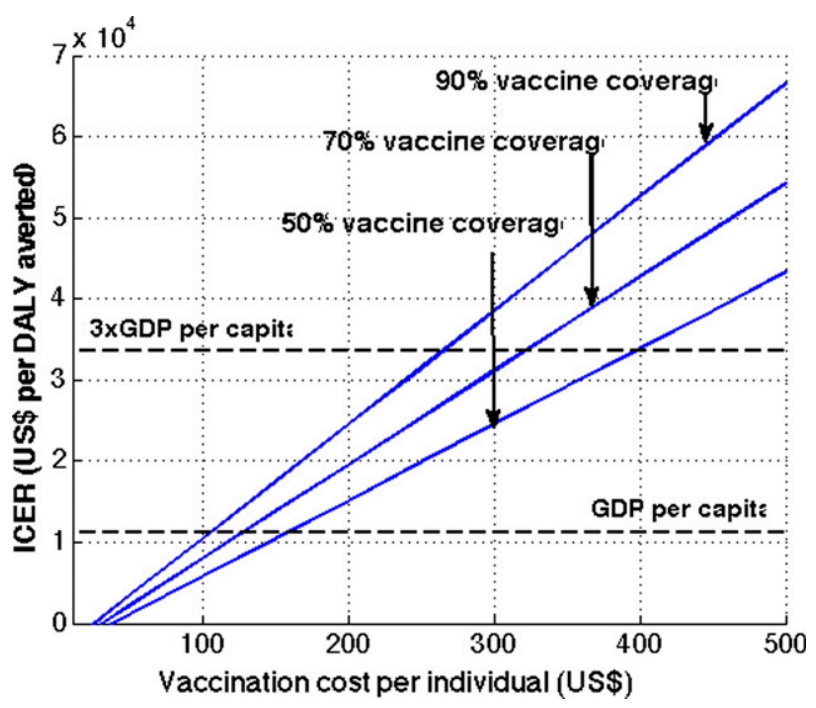

FIGURE 5. Cost-effectiveness of routine dengue vaccination with various coverage levels. The routine dengue vaccination of 9 -year-olds was considered with $50 \%, 70 \%$, and $90 \%$ vaccine coverage levels.

\section{DISCUSSION}

The first dengue vaccine has now been approved for use in 13 countries. However, the impact of the new dengue vaccine might be hampered by the risk of immune-mediated enhancement of disease. Specifically, the trials of CYD-TDV revealed much lower efficacy in recipients who were seronegative at the time of vaccination than in those who were seropositive to dengue virus at the time of vaccination. As a result, lower vaccine efficacies were observed in younger age groups that have not lived long enough to experience a natural infection.

Even with the relatively low vaccine efficacy estimated in the recent dengue vaccine trials, our results show that age-targeted vaccination may still be cost-effective in Brazil. We show that routine vaccination of $70 \%$ of 9 -year-olds would reduce the incidence of dengue infection by $79 \%$ and would be cost-effective across a range of vaccination costs.

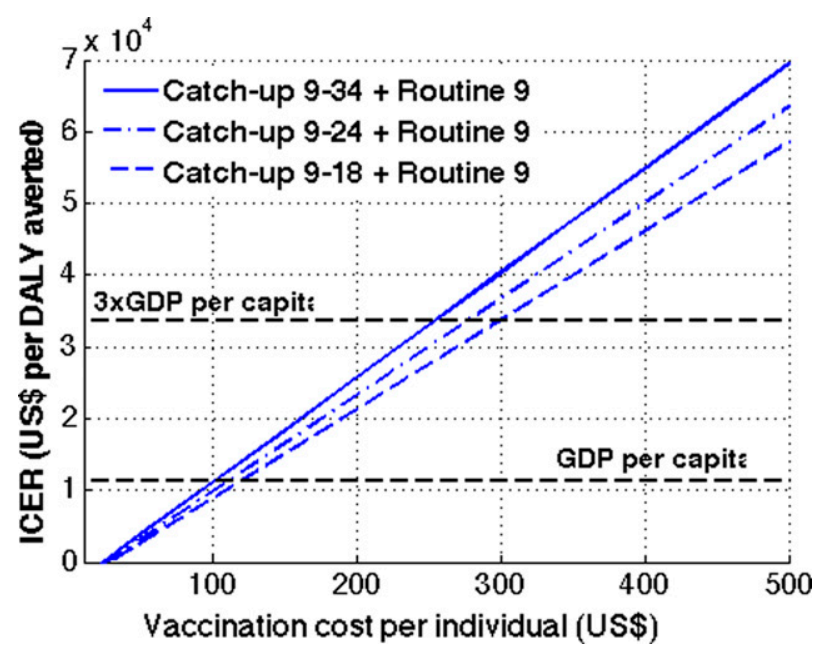

FIGURE 6. Cost-effectiveness of a catch-up campaign followed by routine dengue vaccination. One year of a catch-up campaign with various target ages (9-18, 9-24, and 9-34) followed by routine dengue vaccination of 9-year-olds was considered. For catch-up campaigns, $50 \%$ vaccine coverage was assumed, whereas $70 \%$ vaccine coverage was used for routine vaccination. 
Achieving this level of vaccination coverage is expected to be feasible, given the high levels of adherence to childhood vaccination schedules in Brazil. Routine vaccination policies with higher vaccine coverage levels $(90 \%)$ would result in higher cost-effectiveness ratios, but would be still considered cost-effective as long as the cost per course of vaccination is under \$262. Similarly, for vaccination policies with a catch-up campaign, the cost-effectiveness of which was shown to be dependent on the targeted ages. If wider target ages were selected for a catch-up campaign, the cost-effectiveness of the vaccination program would be reduced, because the expansion of coverage would have the greatest health impact at the lowest coverage due to the decreasing marginal returns of herd immunity. Nevertheless, policies that combine routine coverage with onetime mass vaccination of vaccine-eligible age groups (i.e., ages 9-45) would have the highest impact of reducing the incidence of both DF and DHF by minimizing the lag time before population immunity is established.

Although our model incorporates some of key features of the newly developed dengue vaccine, such as vaccineinduced ADE and reduced efficacy in seronegative recipients, our analysis also has several limitations. In our model, serotype-specific efficacy parameters were not estimated. This is because country- and serotype-specific trial data are not currently publicly available; these data are necessary to estimate serotype-specific vaccine efficacy by fitting the observed serotype-specific attack rates. ${ }^{8,42}$ Second, outcomes measured in the dengue vaccine trials were based on clinically apparent disease, so it is currently unclear whether the vaccine protects against infection or just against disease. ${ }^{56}$ Our model assumes that both effects of the vaccine occur, that is, protection against infection as well as reduction in disease risk. Future expansions of our model could investigate the effects of various vaccine mechanisms on population protection and on the cost-effectiveness of a vaccination program. Furthermore, the development of agent-based model of dengue transmission and its use in the cost-effectiveness analysis would capture the spatial and temporal heterogeneity.

The incidence of dengue and its dispersion are rising due to climate changes, population growth, urbanization, and globalization. We evaluated the cost-effectiveness of various vaccination strategies in Brazil in light of the recent dengue vaccine approval. We show that carefully targeted vaccination would be cost-effective as a prevention strategy in Brazil and holds a potential to reduce the overall epidemiological and economic burden of dengue in the country.

Received October 15, 2016. Accepted for publication January 26, 2017.

Published online March 6, 2017.

Note: Supplemental information and figure appear at www.ajtmh.org.

Financial support: This research was supported by the Basic Science Research Program through the National Research Foundation of Korea (NRF) funded by the Ministry of Education (2015R1D1A1A01058208).

Author's address: Eunha Shim, Department of Mathematics, Soongsil University, 369 Sangdo-Ro, Dongjak-Gu, Seoul, Republic of Korea, 06978. E-mail: alicia@ssu.ac.kr.

This is an open-access article distributed under the terms of the Creative Commons Attribution License, which permits unrestricted use, distribution, and reproduction in any medium, provided the original author and source are credited.

\section{REFERENCES}

1. Fares RC, Souza KP, Anez G, Rios M, 2015. Epidemiological scenario of dengue in Brazil. BioMed Res Int 2015: 321873.

2. World Health Organization, 2009. Dengue: Guidelines for Diagnosis, Treatment, Prevention and Control: New Edition. Geneva, Switzerland: World Health Organization.

3. Bhatt S, Gething PW, Brady OJ, Messina JP, Farlow AW, Moyes CL, Drake JM, Brownstein JS, Hoen AG, Sankoh O, Myers MF, George DB, Jaenisch T, Wint GR, Simmons CP, Scott TW, Farrar JJ, Hay SI, 2013. The global distribution and burden of dengue. Nature 496: 504-507.

4. Halstead SB, 1988. Pathogenesis of dengue: challenges to molecular biology. Science 239: 476-481.

5. Endy TP, Nisalak A, Chunsuttitwat S, Vaughn DW, Green S, Ennis FA, Rothman AL, Libraty DH, 2004. Relationship of preexisting dengue virus (DV) neutralizing antibody levels to viremia and severity of disease in a prospective cohort study of DV infection in Thailand. J Infect Dis 189: 990-1000.

6. Halstead SB, 1982. Immune enhancement of viral infection. Prog Allergy 31: 301-364.

7. World Health Organization, 2017. Dengue vaccine: WHO position paper, July 2016 - recommendations. Vaccine 35: 1200-1201.

8. Ferguson NM, Rodríguez-Barraquer I, Dorigatti I, Mier-y-TeranRomero L, Laydon DJ, Cummings DA, 2016. Benefits and risks of the Sanofi-Pasteur dengue vaccine: modeling optimal deployment. Science 353: 1033-1036.

9. Guy B, Guirakhoo F, Barban V, Higgs S, Monath TP, Lang J, 2010. Preclinical and clinical development of YFV 17D-based chimeric vaccines against dengue, West Nile and Japanese encephalitis viruses. Vaccine 28: 632-649.

10. Capeding MR, Tran NH, Hadinegoro SR, Ismail HI, Chotpitayasunondh T, Chua MN, Luong CQ, Rusmil K, Wirawan DN, Nallusamy R, Pitisuttithum P, Thisyakorn U, Yoon IK, van der Vliet D, Langevin E, Laot T, Hutagalung $Y$, Frago C, Boaz M, Wartel TA, Tornieporth NG, Saville M, Bouckenooghe A, Group CYDS, 2014. Clinical efficacy and safety of a novel tetravalent dengue vaccine in healthy children in Asia: a phase 3, randomised, observer-masked, placebocontrolled trial. Lancet 384: 1358-1365.

11. Villar L, Dayan GH, Arredondo-Garcia JL, Rivera DM, Cunha R, Deseda C, Reynales H, Costa MS, Morales-Ramirez JO, Carrasquilla G, Rey LC, Dietze R, Luz K, Rivas E, Montoya MC, Supelano MC, Zambrano B, Langevin E, Boaz M, Tornieporth N, Saville M, Noriega F; CYD15 Study Group, 2015. Efficacy of a tetravalent dengue vaccine in children in Latin America. N Engl J Med 372: 113-123.

12. Hadinegoro SR, Arredondo-Garcia JL, Capeding MR, Deseda $\mathrm{C}$, Chotpitayasunondh $\mathrm{T}$, Dietze R, Muhammad Ismail $\mathrm{HI}$, Reynales H, Limkittikul K, Rivera-Medina DM, Tran HN, Bouckenooghe A, Chansinghakul D, Cortes M, Fanouillere K, Forrat R, Frago C, Gailhardou S, Jackson N, Noriega F, Plennevaux E, Wartel TA, Zambrano B, Saville M; CYD-TDV Dengue Vaccine Working Group, 2015. Efficacy and long-term safety of a dengue vaccine in regions of endemic disease. N Engl J Med 373: 1195-1206.

13. Hladish TJ, Pearson CA, Chao DL, Rojas DP, Recchia GL, Gomez-Dantes H, Halloran ME, Pulliam JR, Longini IM, 2016. Projected impact of dengue vaccination in Yucatan, Mexico. PLoS Negl Trop Dis 10: e0004661.

14. Aguiar M, Stollenwerk N, Halstead SB, 2016. The risks behind Dengvaxia recommendation. Lancet Infect Dis 16: 882-883.

15. Shepard DS, Suaya JA, Halstead SB, Nathan MB, Gubler DJ, Mahoney RT, Wang DN, Meltzer MI, 2004. Cost-effectiveness of a pediatric dengue vaccine. Vaccine 22: 1275-1280.

16. Lee BY, Connor DL, Kitchen SB, Bacon KM, Shah M, Brown ST, Bailey RR, Laosiritaworn Y, Burke DS, Cummings DA, 2011. Economic value of dengue vaccine in Thailand. $A m ~ J$ Trop Med Hyg 84: 764-772.

17. Carrasco LR, Lee LK, Lee VJ, Ooi EE, Shepard DS, Thein TL, Gan V, Cook AR, Lye D, Ng LC, Leo YS, 2011. Economic impact of dengue illness and the cost-effectiveness of future vaccination programs in Singapore. PLoS Negl Trop Dis 5: e1426. 
18. Durham DP, Ndeffo Mbah ML, Medlock J, Luz PM, Meyers LA, Paltiel AD, Galvani AP, 2013. Dengue dynamics and vaccine cost-effectiveness in Brazil. Vaccine 31: 3957-3961.

19. Orellano PW, Reynoso JI, Stahl HC, Salomon OD, 2016. Costutility analysis of dengue vaccination in a country with heterogeneous risk of dengue transmission. Vaccine 34: 616-621.

20. Flasche S, Jit M, Rodriguez-Barraquer I, Coudeville L, Recker M, Koelle K, Milne G, Hladish TJ, Perkins TA, Cummings DA, Dorigatti I, Laydon DJ, Espana G, Kelso J, Longini I, Lourenco J, Pearson CA, Reiner RC, Mier YT-RL, Vannice K, Ferguson $\mathrm{N}, 2016$. The long-term safety, public health impact, and cost-effectiveness of routine vaccination with a recombinant, live-attenuated dengue vaccine (Dengvaxia): a model comparison study. PLoS Med 13: e1002181.

21. Shim E, 2016. Dengue dynamics and vaccine cost-effectiveness analysis in the Philippines. Am J Trop Med Hyg 95: 1137-1147.

22. Shepard DS, Undurraga EA, Halasa YA, Stanaway JD, 2016. The global economic burden of dengue: a systematic analysis. Lancet Infect Dis 16: 935-941.

23. Shim E, 2016. Dengue dynamics and vaccine cost-effectiveness analysis in the Philippines. Am J Trop Med Hyg 95: 1137-1147.

24. Halstead SB, Russell PK, 2016. Protective and immunological behavior of chimeric yellow fever dengue vaccine. Vaccine 34: 1643-1647.

25. Gibbons RV, Kalanarooj S, Jarman RG, Nisalak A, Vaughn DW, Endy TP, Mammen MP Jr, Srikiatkhachorn A, 2007. Analysis of repeat hospital admissions for dengue to estimate the frequency of third or fourth dengue infections resulting in admissions and dengue hemorrhagic fever, and serotype sequences. Am J Trop Med Hyg 77: 910-913.

26. Olkowski S, Forshey BM, Morrison AC, Rocha C, Vilcarromero S, Halsey ES, Kochel TJ, Scott TW, Stoddard ST, 2013. Reduced risk of disease during postsecondary dengue virus infections. J Infect Dis 208: 1026-1033.

27. Pandey A, Medlock J, 2015. The introduction of dengue vaccine may temporarily cause large spikes in prevalence. Epidemiol Infect 143: 1276-1286.

28. Halstead SB, 2003. Neutralization and antibody-dependent enhancement of dengue viruses. Adv Virus Res 60: 421-467.

29. Nagao Y, Koelle K, 2008. Decreases in dengue transmission may act to increase the incidence of dengue hemorrhagic fever. Proc Natl Acad Sci USA 105: 2238-2243.

30. Adams B, Boots M, 2006. Modelling the relationship between antibody-dependent enhancement and immunological distance with application to dengue. J Theor Biol 242: 337-346.

31. Edillo FE, Halasa YA, Largo FM, Erasmo JN, Amoin NB, Alera MT, Yoon IK, Alcantara AC, Shepard DS, 2015. Economic cost and burden of dengue in the Philippines. Am J Trop Med Hyg 92: 360-366.

32. Cordeiro MT, Silva AM, Brito CA, Nascimento EJ, Magalhaes MC, Guimaraes GF, Lucena-Silva N, de Carvalho EM, Marques ET Jr, 2007. Characterization of a dengue patient cohort in Recife, Brazil. Am J Trop Med Hyg 77: 1128-1134.

33. Shepard DS, Coudeville L, Halasa YA, Zambrano B, Dayan GH, 2011. Economic impact of dengue illness in the Americas. Am J Trop Med Hyg 84: 200-207.

34. Araujo DV, Bahia L, Rendeiro M, Pinho AS, Genovez V, de Menezes Gonçalves T, Abreu M, 2016. Estimation of the potential impact of dengue vaccination on clinical outcomes in Brazil. Journal Brasileiro de Economia da Saúde 8: 3-15.

35. Ndeffo Mbah ML, Durham DP, Medlock J, Galvani AP, 2014. Country-and age-specific optimal allocation of dengue vaccines. J Theor Biol 342: 15-22.

36. PAHO, 2010. Vaccine Introduction Guidelines 2010. Available at: http://new.paho.org/hq/dmdocuments/2010/FieldGuide_ NewVaccines_1stEd_e.pdf. Accessed August 30, 2016.
37. Luz PM, Vanni T, Medlock J, Paltiel AD, Galvani AP, 2011. Dengue vector control strategies in an urban setting: an economic modelling assessment. Lancet 377: 1673-1680.

38. Von Allmen SD, Lopez-Correa RH, Woodall JP, Morens DM, Chiriboga J, Casta-Velez A, 1979. Epidemic dengue fever in Puerto Rico, 1977: a cost analysis. Am J Trop Med Hyg 28: $1040-1044$.

39. Johansson MA, Hombach J, Cummings DA, 2011. Models of the impact of dengue vaccines: a review of current research and potential approaches. Vaccine 29: 5860-5868.

40. Gubler DJ, 1998. Dengue and dengue hemorrhagic fever. Clin Microbiol Rev 11: 480-496.

41. Guy B, Jackson N, 2016. Dengue vaccine: hypotheses to understand CYD-TDV-induced protection. Nat Rev Microbiol 14: $45-54$

42. Coudeville L, Baurin N, Vergu E, 2016. Estimation of parameters related to vaccine efficacy and dengue transmission from two large phase III studies. Vaccine 34: 6417-6425.

43. Martelli CM, Siqueira JB Jr, Parente MP, Zara AL, Oliveira CS, Braga C, Pimenta FG Jr, Cortes F, Lopez JG, Bahia LR, Mendes MC, da Rosa MQ, de Siqueira Filha NT, Constenla D, de Souza WV, 2015. Economic impact of dengue: multicenter study across four Brazilian regions. PLoS Negl Trop Dis 9: e0004042.

44. Murray CJ, 1994. Quantifying the burden of disease: the technical basis for disability-adjusted life years. Bull World Health Organ 72: 429-445.

45. Dantes HG, Farfan-Ale JA, Sarti E, 2014. Epidemiological trends of dengue disease in Mexico (2000-2011): a systematic literature search and analysis. PLoS Negl Trop Dis 8: e3158.

46. Chao DL, Halstead SB, Halloran ME, Longini IM Jr, 2012. Controlling dengue with vaccines in Thailand. PLoS Negl Trop Dis 6: e1876.

47. Rodriguez-Barraquer I, Mier-y-Teran-Romero L, Schwartz IB, Burke DS, Cummings DA, 2014. Potential opportunities and perils of imperfect dengue vaccines. Vaccine 32: 514-520.

48. Anderson KB, Chunsuttiwat S, Nisalak A, Mammen MP, Libraty DH, Rothman AL, Green S, Vaughn DW, Ennis FA, Endy TP, 2007. Burden of symptomatic dengue infection in children at primary school in Thailand: a prospective study. Lancet 369: 1452-1459.

49. Smith KJ, Raviotta JM, DePasse JV, Brown ST, Shim E, Patricia Nowalk M, Zimmerman RK, 2016. Cost effectiveness of influenza vaccine choices in children aged 2-8 years in the U.S. Am J Prev Med 50: 600-608.

50. Goldie SJ, Kim JJ, Kobus K, Goldhaber-Fiebert JD, Salomon J, O'Shea MK, Xavier Bosch F, de Sanjose S, Franco EL, 2007. Cost-effectiveness of HPV 16, 18 vaccination in Brazil. Vaccine 25: 6257-6270.

51. Shim E, Galvani AP, 2009. Impact of transmission dynamics on the cost-effectiveness of rotavirus vaccination. Vaccine 27 : 4025-4030.

52. United States Department of Labor, 2007. The Consumer Price Indexes (CPI): All Urban Consumers. Available at: http:// stats.bls.gov/cpi/home.htm. Accessed November 26, 2007.

53. CIA, 2017. The World Factbook-Brazil. Available at: https:// www.cia.gov/library/publications/the-world-factbook/geos/ br.html. Accessed August 30, 2016.

54. WHO, 2002. World Health Report 2002: Reducing Risks, Promoting Healthy Life, Report of the Commission on Macroeconomics and Health. Geneva, Switzerland: World Health Organization.

55. The World Bank, 2016. GDP Per Capita. Available at: http:// data.worldbank.org/indicator/NY.GDP.PCAP.CD?locations=BR. Accessed August 30, 2016.

56. Rodriguez-Barraquer I, Mier-y-Teran-Romero L, Burke DS, Cummings DA, 2013. Challenges in the interpretation of dengue vaccine trial results. PLoS Negl Trop Dis 7: e2126. 